\title{
THE KINEMATICS AND ABUNDANCES OF STAR CLUSTERS IN THE LARGE MAGELLANIC CLOUD
}

\author{
R.A. SCHOMMER \\ Cerro Tololo Inter-American Observatory \\ Casilla 603 \\ La Serena \\ Chile
}

\begin{abstract}
We present velocities for 75 star clusters in the Large Magellanic Cloud (LMC), based on individual star measures at the Calcium triplet The spectra are also used to derive abundances for most of the clusters, and an age-metallicity relation is presented for the inner and outer regions. One half of the clusters are objects in the outer parts of the LMC (radius $\geq 5^{\circ}$ ), which had no previous velocity determinations. Published velocities for intermediate and old clusters are shown to have had systematic errors. We compare these velocities with various rotation curve analyses of the LMC, and test aspects of the twisted disk model of Freeman et al. (1983, hereafter FIO). Our outer cluster sample and the intermediate age inner clusters form a disk which agrees with the parameters of the optical isophotes and inner HI rotation curve. The oldest clusters are still enigmatic; they appear to be flattened and rotate much as FIO have described, although the parameters are poorly determined in this small sample.
\end{abstract}

\section{Introduction}

The star clusters in the Magellanic Clouds provide a rich resource for studying aspects of stellar and chemical evolution, star formation history, and internal and system dynamics. Over the past several years, we have been investigating clusters in the outer parts of the LMC (Olszewski et al. 1988, OHSC).

I report here on abundances and kinematics of individual stars in a sample of 50 clusters from OHSC ( 36 with radius $\geq 5^{\circ}$ ), which also includes the Mateo (1988) field. In general, the ages for these clusters are unknown, although Olszewski (1988) finds ages of 2-3 Gyr for six clusters, and published colour-magnitude diagrams generally yield similar intermediate ages. We also reobserved all the FIO clusters of SWB type V-VII (Searle et al. 1980).

\section{Abundances}

The spectra were obtained with the CTIO $4 \mathrm{~m}$ telescope, in the Calcium triplet region (8500$8700 \AA)$. Equivalent widths were measured to an accuracy of $0.3 \AA$ ( $( \pm 0.2 \mathrm{dex})$. Plots of equivalent widths (the sum of all three $\mathrm{Ca}$ lines) versus magnitude show considerable sensitivity to abundance, and Fig. 1 shows our data for the LMC cluster stars on such a diagram. Also shown are lines derived from our observations of stars in a sample of Galactic globular and open clusters to provide an abundance calibration; the metallicity scale used was that of Zinn (1985). A similar calibration has been found by Da Costa (1988). 
From Fig. 1 we conclude that all old clusters (SWB VII) are metal-poor. Examples are NGC 1466, $(\mathrm{m}(\mathrm{Ca})=-2.2)$, NGC $1835(-1.8)$, NGC $2210(-2.0)$, and Hodge 11(-2.1). Essentially, all the new clusters are moderately metal-rich, between 47 Tuc and solar metallicities; most of the SWB IV-VI clusters also lie in this range. E121SC03 (see discussion below) is plotted as an open circle in Fig. 1. We also find that all clusters more metal-poor than -1.3 in the sample were already known as SWB VII clusters. Thus none of these new clusters is likely to be an extreme halo object like the SWB VII clusters.

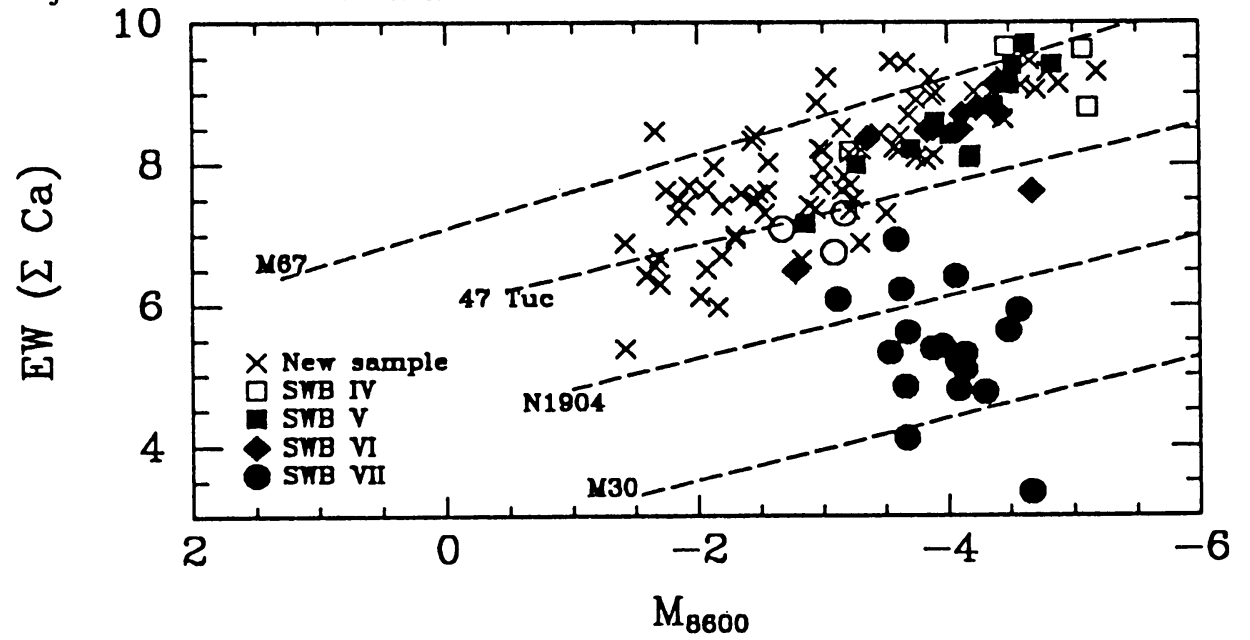

Figure 1. Metallicities of individual stars in LMC clusters.

For a sample of our clusters (including many of the FIO objects) ages have been determined from colour-magnitude diagrams, and we present the age-metallicity relation (AMR) for this subsample in Fig. 2. The line from Cohen (1982), which represented a simple one-zone enrichment model for her data, is shown, and represents our data well. Mateo (1988) also found a similar AMR for his field. Somewhat to our surprise the mean metallicity for the outer clusters is quite close to that of the inner objects. At $1 \mathrm{Gyr}$, the outer clusters have a mean metallicity of -0.42 , and the inner clusters yield -0.29 , with the scatter of \pm 0.2 dex mostly attributable to observational error. Considerable enrichment has occurred in the outer regions of the LMC, although current HI density in these regions is only $\sim 5 \times 10^{19} \mathrm{~cm}^{-2}$. (One cluster, NGC1754, a filled square in Fig. 2 , is far away from the mean AMR. We suspect that field stars may have contaminated the age determination in this case.)

One striking aspect in Fig. 2 is the gap in both metallicity (from -1.7 to -0.7) and age (from 3$12 \mathrm{Gyr}$ ) which is filled by only one cluster, E121SC03 (Mateo et al. 1986), at $10 \mathrm{Gyr}$ and -0.9 dex (the open circles in Fig. 1). Finding clusters in this age and abundance gap has been difficult, but such objects are very important for tracing the cluster formation and chemical enrichment history of the LMC. Clusters 33 and 37 in the OHSC list have abundances near -1 and are possible transition objects.

\section{Velocities of the cluster system}

Our cluster velocities are measured by cross correlation techniques against standard stars and the mean error of a single measure is $\pm 5 \mathrm{~km} \mathrm{~s}^{-1}$. Most clusters have two or more stars measured. 


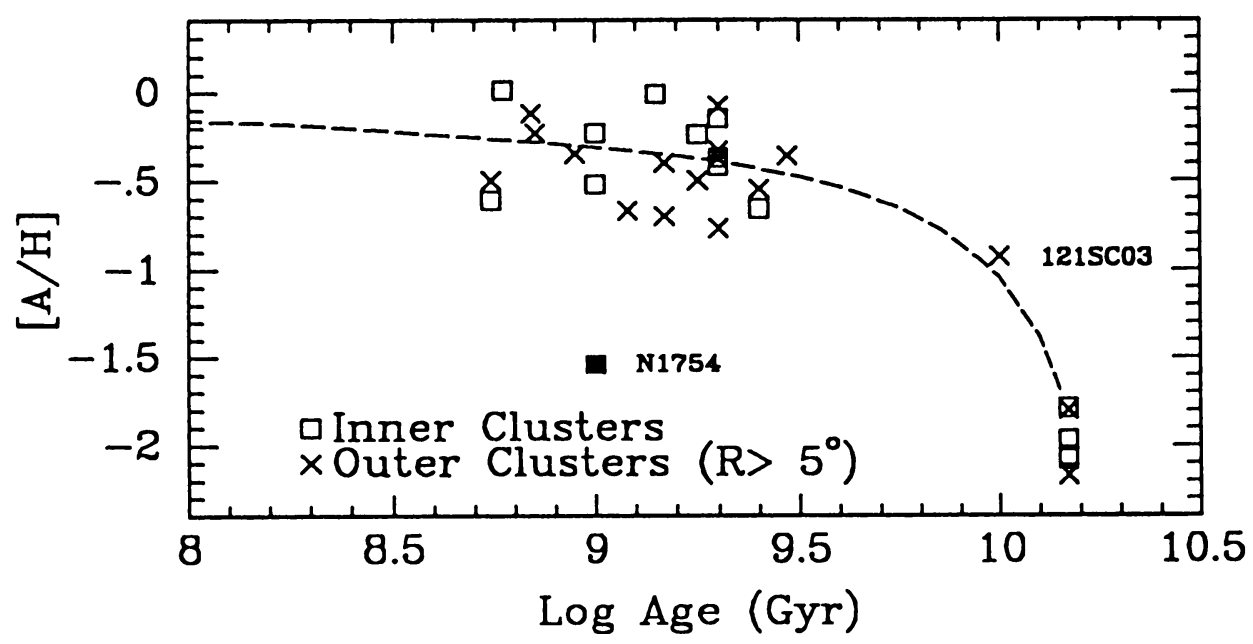

Figure 2. The age-metallicity relation for the cluster sample.

Kinematical analyses of stellar systems over the past several years have focused on aspects of the FIO results and needs a brief summary here. FIO found that the young LMC clusters (SWB I-III) rotated with the HI gas, but that the intermediate ages and old clusters (SWB V-VII) showed peculiar and distinct properties. The three distinctive properties were: 1) a rotating, flattened system, with a small z-dispersion; 2) the major axis of this rotation was twisted by nearly $50^{\circ}$ with respect to the isophotal position angle and the young disk; and 3) the systemic velocity of this old cluster system was lower by ab out $18 \mathrm{~km} \mathrm{~s}^{-1}$ than the $\mathrm{HI}$ or young clusters.

Our velocities permit us to explore the kinematics of a larger group of clusters, many in the outer parts of the LMC, where twists, due to the interaction between the Magellanic clouds and the Galaxy, might be significant. Several FIO clusters were included in our original program; we found velocities for these systems to be different from the FIO compilation (which were based upon integrated spectra from several sources) and so we reobserved all SWB V-VII clusters in the FIO sample.

Table 1 presents a summary of the kinematic solutions we have found. The first two solutions listed are the for the HI (and young disk) and for the old cluster system, from the FIO paper. Solution 3 is our first fit for the new sample. We find a systemic velocity that agrees well with the young objects, but an intermediate position angle. Solution 4 represents the FIO old cluster sample, but with our new velocities. We still find sizeable rotation in this cluster system, with a different position angle from the young disk. Our first conclusion from these fits is that one part of the puzzle seems to have been solved: the systemic velocities now agree within for all clusters. A systematic error was present in the FIO velocities, of about $18 \mathrm{~km} \mathrm{~s}^{-1}$, which led to that curious result.

Much of our sample lies at a sufficiently large distance from the LMC centre that the transverse component of the LMC must be taken into account. The effects of this motion are well known to cause an apparent increase in the PA of the major axis (e.g. Feitzinger et al. 1977, Prevot et al. 1989). While the dynamical determination of this transverse motion makes strong assumptions about the uniformity of the LMC rotation, preliminary measures of the proper motion of the LMC (Jones et al. 1989) confirm a motion of order $200 \mathrm{~km} \mathrm{~s}^{-1}$ eastward. We have therefore computed solutions to correct for this term, taking $V_{t}=150 \mathrm{~km} \mathrm{~s}^{-1}$ (solutions 5-8). Solutions with $\mathrm{V}_{t}=250 \mathrm{~km} \mathrm{~s}^{-1}$ (solution $5^{\prime}$ ) are also acceptable. 
Table 1. Rotation solutions

\begin{tabular}{clcllccl} 
Sol.\# & Objects & $\mathrm{N}$ & $\mathrm{V}_{\text {circ }}$ & $\Theta(\mathrm{PA})$ & $\mathrm{Vsys}$ & $\sigma$ & Comments \\
\hline 1 & FIO HI & $\ldots$ & 79 & -9 & 44 & $\ldots$ & FIO HI solution \\
2 & FIO old (V-VII) & 25 & $90 \pm 11$ & $41 \pm 5$ & $26 \pm 2$ & 18 & FIO old soln\#3 \\
& & & & & & & \\
3 & New Clusters & 50 & $71 \pm 8$ & $14 \pm 8$ & $44 \pm 3$ & 19 & mostly outer \\
4 & SWB V-VII & 28 & $72 \pm 15$ & $31 \pm 10$ & $39 \pm 4$ & 21 & FIO, new vels \\
& & & & & & & \\
5 & New Clusters & 50 & $69 \pm 8$ & $-7 \pm 10$ & $42 \pm 3$ & 17 & V $_{t}=150 \mathrm{~km} / \mathrm{sec}$ \\
5 & New Clusters & 50 & $73 \pm 9$ & $-20 \pm 10$ & $42 \pm 3$ & 16 & V $_{t}=250 \mathrm{~km} / \mathrm{sec}$ \\
6 & SWB V-VI & 17 & $54 \pm 14$ & $-7 \pm 14$ & $36 \pm 5$ & 17 & intermediate age \\
7 & SWB VII & 10 & $67 \pm 27$ & $37 \pm 25$ & $38 \pm 6$ & 24 & oldest \\
8 & SWB VII & 13 & $67 \pm 22$ & $35 \pm 17$ & $38 \pm 5$ & 21 & old + N1841... \\
\hline
\end{tabular}

The new (outer) cluster sample is now found to rotate with parameters essentially identical to the young (inner) disk. The fits for these solutions $(5,6,8)$ are plotted in Fig. 3. The velocity dispersion we find for the new cluster sample about the rotation fit is similar to the value we derive for a dispersion in a local region of clusters. For example, the dispersion for 18 clusters in a southern "spiral arm" region is $16 \mathrm{~km} \mathrm{~s}^{-1}$. This dispersion translates into a $z$ scale height of $\sim 0.5$ kpc.

We now find, moreover, that if we break the FIO "old sample" into intermediate age (SWB V-VI or IV-VI) and an oldest bin (SWB VII), solutions 6 and 7 respectively, the intermediate sample rotates in a manner similar to the HI disk. The amplitude of the rotation is somewhat smaller, but the disks appear to have the same orientation. Of course, in our own Galaxy only the clusters older than $10 \mathrm{Gyr}$ seem to correspond to a true halo component. The translation of integrated properties like SWB type into age is an exacting task, but all the clusters believed to be old (i.e. $t \geq 10 \mathrm{Gyr}$ ) fall into SWB class VII. There still may be some intermediate age interlopers in this bin, but at least half the objects have colour-magnitude diagrams that indicate large ages, or have detected RR Lyrae. Therefore, a search for a "kinematic halo" should separate out the obvious intermediate age contamination.

Even with this distinction, the oldest sample (SWB VII) still gives an unusual position angle for the line of nodes (twisted), some $40^{\circ}$ different from the HI disk. The PA is rather poorly determined by this small sample, and the plots give one little faith that the rotation of this component is well understood. Several of the clusters (e.g. N2210, H11) lie $\geq 45 \mathrm{~km} \mathrm{~s}^{-1}$ from the best fit disk or the $\mathrm{HI}$ solution. It is probably impossible to tell from such a small sample whether the LMC has a kinematic halo. The velocity dispersion is still quite low $\left(\sim 20-25 \mathrm{~km} \mathrm{~s}^{-1}\right)$, while a true kinematical halo would require a disperson of $79 / \sqrt{3} \sim 46 \mathrm{~km} \mathrm{~s}^{-1}$. The eight "best case" SWB VII clusters do give a dispersion of about $44 \mathrm{~km} \mathrm{~s}^{-1}$ about the mean (i.e. assuming no rotation). Thus an old halo with this dispersion still could exist, but velocities of a larger sample of unambiguously old objects (e.g. metal-poor field RR Lyrae) would be required to detect it with confidence.

We have attempted to supplement this old sample by adding velocities for some remote, old clusters, including the Reticulum system $\left(\mathrm{V}_{h}=+243 \mathrm{~km} \mathrm{~s}^{-1}\right.$;radius $\left.=11.4^{\circ}\right)$, E121SC03 $(+309$ $\mathrm{km} \mathrm{s}^{-1} ; 9.7^{\circ}$ ), and NGC $1841\left(+214 \mathrm{~km} \mathrm{~s}^{-1} ; 1^{\circ} .9^{\circ}\right)$ (Suntzeff et al. in preparation). These 


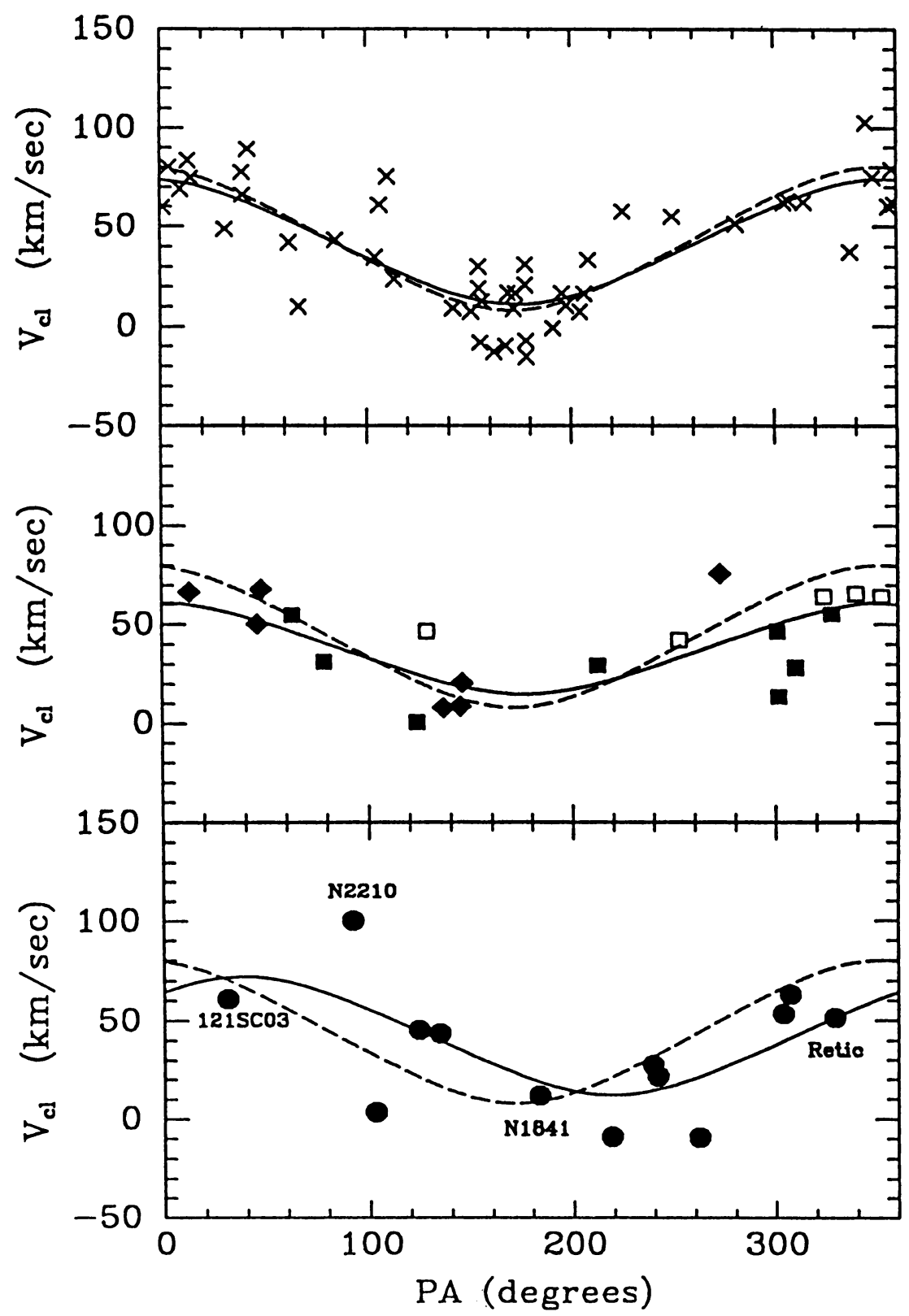

Figure 3. Velocity $\mathbf{v}$ position angle for cluster samples. Symbols are as in Fig. 1 The dashed line in all frames is the HI rotation solution, the solid line the fit to the cluster samples, corrected for transverse motion. Top: New clusters; Centre: FIO IV-VI clusters; Bottom: FIO VII clusters. 
clusters fall on the rotation curve for the old clusters (Fig. 3, bottom), and would seem to imply a flat rotation curve out to $10^{\circ}(8 \mathrm{kpc})$ or more for this old cluster population.

Much of the work described here is a result of labours by Ed Olszewski, Nick Suntzeff, and Hugh Harris. I am indebted to them for their insights as well as hard work. I thank Dave Hartwick and Ken Freeman for enlightening conversations, and the CTIO TAC for $4 \mathrm{~m}$ allocations.

\section{References}

Cohen, J. (1982), Ap. J. 258, 143.

Da Costa, G.S. (1988), IAU Symp. 126 Globular Cluster Systems in Galaxies, J.E. Grindlay, A.G.C. Philip (eds.) (Reidel: Dordrecht) p.217.

Feitzinger, J., Isserstedt, J., Schmidt-Kahler, Th. (1977), Astron. Astrophys. 57, 265.

Freeman, K.C., Illingworth, G., Oemler, A. (1983), Ap. J. 272, 488. (FIO).

Jones, B., Klemola, A., Lin, D.C. (1989), Bull. A.A.S. 21, 1107.

Mateo, M. (1988), IAU Symp. 126 Globular Cluster Systems in Galaxies, J.E. Grindlay, A.G.C. Philip (eds.) (Reidel: Dordrecht) p. 557.

Mateo, M., Hodge, P.W., Schommer, R.A. (1986), Ap. J. 311, 113.

Olszewski, E. (1988), IAU Symp. 126 Globular Cluster Systems in Galaxies, J.E. Grindlay, A.G.C. Philip (eds.) (Reidel: Dordrecht) p.159.

Olszewski, E., Harris, H., Schommer, R., Canterna, R. (1988), Astron. Journ. 95, 84.

Prevot, L., Rousseau, J., Martin, N. (1989), Astron. Astrophys. 225, 303.

Searle, L., Wilkinson, A., Bagnuolo, W.G. (1980), Ap. J. 239, 803. (SWB).

Zinn, R., (1985), Ap. J. 293, 424. 